\title{
Confocal Raman AFM, a powerful tool for the nondestructive characterization of heterogeneous materials
}

\author{
U. Schmidt, F. Vargas, M. Kress, K. Weishaupt, and O. Hollricher \\ WITec GmbH, Hoervelsinger Weg 6, Ulm 89081, Germany, www.witec.de
}

The Confocal Raman-AFM is a breakthrough in microscopy. It combines three measuring techniques in one instrument: a high resolution confocal optical microscope, an extremely sensitive Raman spectroscopy system, and an Atomic Force Microscope (AFM). Using this instrument, the high spatial and topographical resolution obtained with an AFM can be directly linked to the chemical information provided by confocal Raman spectroscopy. By simply rotating the microscope turret, the AFM is transformed into a confocal Raman microscope. With the confocal Raman microscope, it is possible to obtain Raman spectra from extremely small sample volumes and to collect high resolution Raman spectral images.

To demonstrate the unique capabilities of the Confocal Raman AFM, an emulsion consisting of alkyd and acrylic latexes has been analyzed. Alkyds and acrylics are used as binders in paints and coatings. These materials produce a shiny, hard finish that is highly water-resistant. Figure 1a shows a 10x10 $\mathrm{m}^{2}$ AFM phase image of a dried alkyd-acrylic latex droplet. Two different phases can be clearly distinguished: a texture less phase, which forms elliptical structures and another phase consisting of spherical particles. The diameter of these particles is approximately $250 \mathrm{~nm}$. Figure $2 \mathrm{~b}$ and c show the Raman spectral images obtained from the same sample area as studied before with the AFM. In the Raman spectral imaging mode, a complete Raman spectrum is acquired at every image pixel, generating a two dimensional array of Raman spectra (multi-spectrum file). The spectral images are obtained by analyzing distinct features of the multi-spectrum file. Integration times of less than $100 \mathrm{~ms} /$ spectrum allow the acquisition of a spectral array of 200x200 spectra in less than one hour, leading to chemical images of the sample with diffraction limited resolution. In the studied emulsion, two characteristic Raman spectra were detected corresponding to the acrylic phase and alkyd (Fig. 2). The distribution of these two phases is visible in the Raman spectral images in Figure $2 \mathrm{~b}$ and $\mathrm{c}$, indicating that the elliptical texture less phase corresponds to alkyd and the spherical fine structure to acrylic latex.

Furthermore, by investigating the tip sample interaction, one can obtain not only the high resolution topographic structure of the surface, but also information about the local mechanical properties of the sample components. Figure 3 shows pulsed force mode (PFM) images of the emulsion. The topography image shows that the spherical acrylic latex particles are surrounded by a chain-like network. The simultaneously recorded adhesion and stiffness images show only a very small difference in adhesion (20\%) between alkyd and acrylic latex and an even smaller stiffness difference of $2 \%$. The detection of such small differences in material properties is only possible if a flexible cantilever with a spring constant below $0.5 \mathrm{~N} / \mathrm{m}$ is used. The phase contrast observed in AC mode (Fig. 1a) can be associated with the stiffness difference measured in pulsed force mode. 

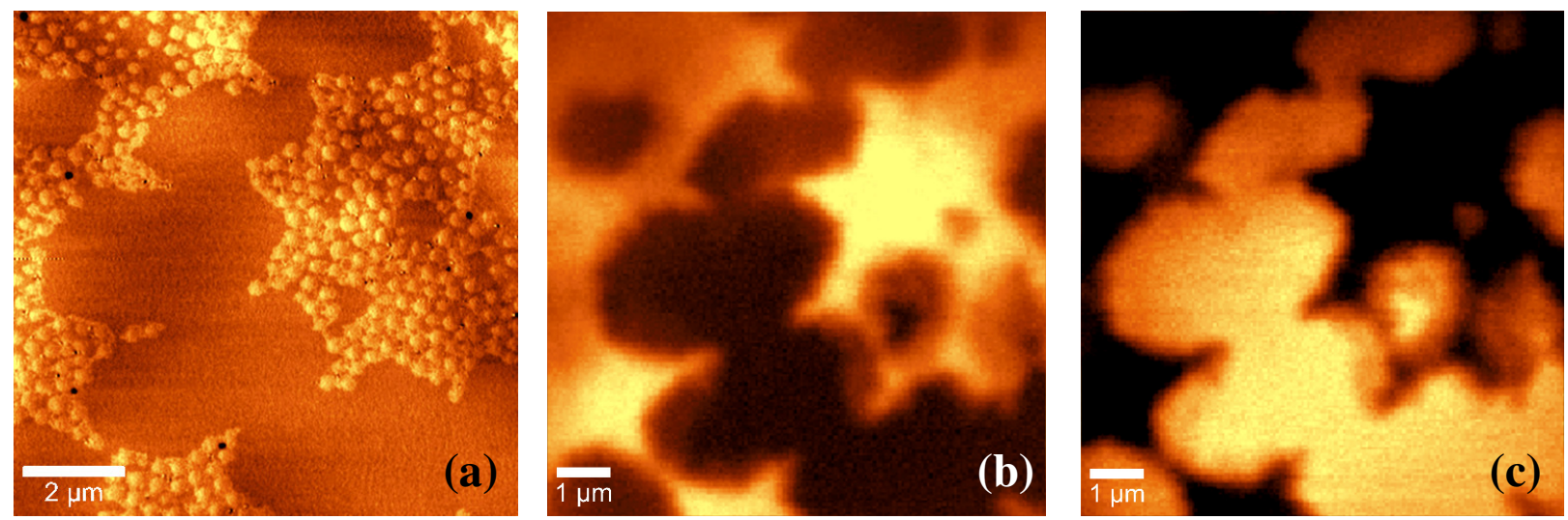

Figure 1: AFM phase image of alkyd-acrylic latex emulsion (a), Raman spectral image of acrylic latex (b), and Raman spectral image of alkyd (c).

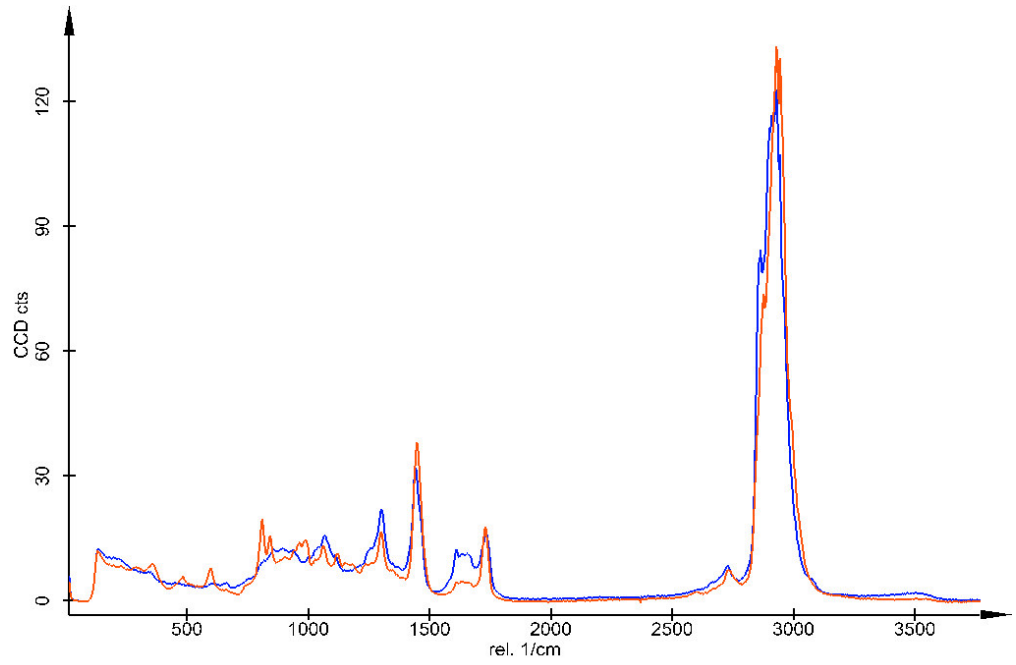

Figure 2: Raman spectra of alkyd (blue) and acrylic latex (red).
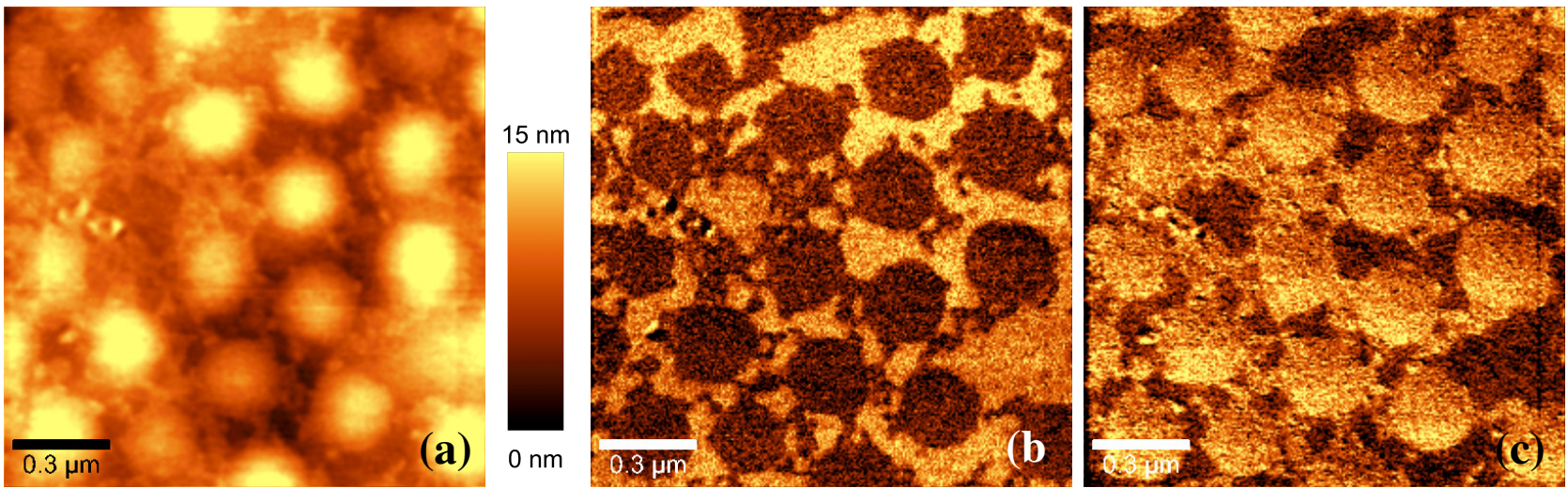

Figure 3: Pulsed fore mode images of the emulsion, topography (a), adhesion (b). and stiffness (c). 